University of New Hampshire

University of New Hampshire Scholars' Repository

Space Science Center

Institute for the Study of Earth, Oceans, and

Space (EOS)

1996

\title{
COMPTEL solar flare measurements
}

James M. Ryan

University of New Hampshire, James.Ryan@unh.edu

Mark L. McConnell

University of New Hampshire - Main Campus, mark.mcconnell@unh.edu

Follow this and additional works at: https://scholars.unh.edu/ssc

Part of the Astrophysics and Astronomy Commons

\section{Recommended Citation}

COMPTEL solar flare measurements Ryan, James M. and McConnell, Mark M., AIP Conference

Proceedings, 374, 200-209 (1996), DOl:http://dx.doi.org/10.1063/1.50956

This Conference Proceeding is brought to you for free and open access by the Institute for the Study of Earth, Oceans, and Space (EOS) at University of New Hampshire Scholars' Repository. It has been accepted for inclusion in Space Science Center by an authorized administrator of University of New Hampshire Scholars' Repository. For more information, please contact Scholarly.Communication@unh.edu. 


\section{AIP | proceedings}

\section{COMPTEL solar flare measurements}

James M. Ryan and Mark M. McConnell

Citation: AIP Conference Proceedings 374, 200 (1996); doi: 10.1063/1.50956

View online: http://dx.doi.org/10.1063/1.50956

View Table of Contents:

http://scitation.aip.org/content/aip/proceeding/aipcp/374?ver=pdfcov

Published by the AIP Publishing

\section{Articles you may be interested in}

Submillimeter/IR solar bursts from high energy electrons

AIP Conf. Proc. 374, 379 (1996); 10.1063/1.50945

Neutron and gammaray measurements of the solar flare of 1991 June 9 AIP Conf. Proc. 294, 89 (1994); 10.1063/1.45205

An overview of solar flare results from COMPTEL AIP Conf. Proc. 294, 21 (1994); 10.1063/1.45193

COMPTEL gamma ray and neutron measurements of solar flares AIP Conf. Proc. 280, 631 (1993); 10.1063/1.44245

Project for geotail observation of the elemental and isotopic abundances of heavy energetic particles in solar flares and galactic cosmic rays AIP Conf. Proc. 183, 390 (1989); 10.1063/1.38016 


\title{
COMPTEL Solar Flare Measurements
}

\author{
James M. Ryan and Mark M. McConnell \\ Space Science Center, University of New Hampshire, Durham, NH 03824
}

\begin{abstract}
We review some of the highlights of the COMPTEL measurements of solar flares. These include images of the Sun in $\gamma$ rays and neutrons. One of the important features of the COMPTEL instrument is its capability to measure weak fluxes of $\gamma$ rays and neutrons in the extended phase of flares. These data complement the spectra taken with the COMPTEL burst spectrometer and the telescope during the impulsive phase of flares. We focus our attention on some of these general capabilities of the instrument and the latest results of two long-duration $\gamma$-ray flares, i.e., 11 and 15 June 1991.
\end{abstract}

\section{INTRODUCTION}

One of the original purposes of double-scatter (now more commonly known as Compton telescopes) was to measure the flux of neutrons arising from the earth's atmosphere as well as the flux of neutrons coming from the Sun. Either or both of these fluxes was postulated to be the origin of the energetic neutron-decay protons in the earth's radiation belts. A series of balloon flights with instruments of this type confirmed that the earth-albedo neutrons could populate the earth's radiation belts. Quiet-Sun measurements of the solar-neutron flux proved negative. Balloon-platform experiments have only short exposures to the Sun, so measuring the active-Sun neutron flux is consequently more difficult than with the same instrument on a spacecraft. However, with the launch of the Compton Gamma Ray Observatory, COMPTEL-a new generation double-scatter telescope-was now capable of fulfilling one of the original objectives of doublescatter telescopes, that of measuring the active-Sun emissions, including both $\gamma$ rays and energetic neutrons. This review of COMPTEL solar data highlights these observations and measurements. A recent review of general high-energy solar-flare phenomena is provided by Hudson and Ryan (1).

Energetic electrons, protons and heavier ions are some of the significant products of the solar-flare process. They can account not only for emissions in generally quiet bands of the electromagnetic spectrum, i.e., microwaves, $\gamma$ rays and 
$\mathrm{X}$ rays, but can also account for a significant fraction of the flare energy. The solar-flare photon emission above $500 \mathrm{keV}$ is particularly revealing. This part of the spectrum contains not only a host of nuclear lines but also the bremsstrahlung emission from relativistic electrons. Beyond $10 \mathrm{MeV}$ lies the part of the spectrum containing radiation from ultrarelativistic electrons and $\pi^{0}$-decay photons at 68 $\mathrm{MeV}$. The decay of charged $\pi$ mesons yields electrons and positrons that in turn radiate. We consider these to be secondary radiations, much like $511 \mathrm{keV}$ annihilation $\gamma$ rays. COMPTEL is sensitive to much of this rich spectrum. In its bulk spectrometer mode it has measured solar $\gamma$ rays from $600 \mathrm{keV}$ to $10 \mathrm{MeV}$ (2), while in its telescope mode it has measured $\gamma$ rays from approximately $800 \mathrm{keV}$ to $30 \mathrm{MeV}$ (3). These measurements complement the concurrent measurements of BATSE (4) and OSSE (generally at lower energies) (5) and EGRET (generally at higher energies) $(6,7)$. Unique to COMPTEL are the measurements of solar flare neutrons-a harkening to its heritage. These neutron measurements further complement the $\gamma$-ray measurements. Together they form a comprehensive set of solar measurements.

The data sample a wide range of the accelerated-particle population in a flare. Nuclear lines are produced by the energetic-proton spectrum in the range of 10-40 $\mathrm{MeV}$, whereas the $\pi^{0}$-decay $\gamma$ rays originate from the proton (or ion) spectrum above about $300 \mathrm{MeV}$. Neutrons in the range of $10-100 \mathrm{MeV}$, on the other hand, sample the proton spectrum at energies slightly higher than the neutrons themselves. The nuclear line at $2.223 \mathrm{MeV}$ from the capture of free neutrons on hydrogen serves as an integral measure of the proton spectrum at all energies above the nuclear binding energy. It is useful as a crude measure of the total energy of the proton population but its origin for any given flare or any moment within a flare is uncertain. The free neutrons could be produced by protons that are also responsible for the nuclear lines but could also be produced by protons that are responsible for the $\pi^{0}$-decay $\gamma$ rays--entirely different parts of the proton spectrum.

This review highlights some of the features and findings of the COMPTEL measurements of solar flares. We illustrate some of the technical properties of the instrument with measurements of so-called long-duration high-energy flares. Some of the more interesting phenomena are associated with these events. The remainder of the paper addresses these relatively rare events and the COMPTEL measurements of them. First, though, we briefly describe the COMPTEL instrument and its features that make it unique in solar-flare studies.

\section{THE COMPTEL INSTRUMENT}

The COMPTEL instrument is described by Schönfelder et al. (8). It is comprised of two detecting systems, D1 and D2. In the detection and measurements of photons, the $\gamma$ rays Compton scatter in D1 and are ideally fully 
absorbed in D2. The locations of the interactions in the detector systems, the energy measurements and the Compton-scatter kinematics restrict the incident $\gamma$ ray direction to be on a circle in the sky that may pass through the location of the Sun. If it does, the measured energy is the full photon energy. A time-of-flight measurement between D1 and D2 serves to greatly improve the signal-to-noise ratio. This time-of-flight measurement is employed in the measurement of neutrons in the range of 10-100 MeV. Instead of Compton scattering in D1, the neutron elastically scatters off hydrogen in the scintillator material in D1. The scattered neutron-now with less energy-is detected in D2. The time-of-flight measurement is equivalent to measuring the scattered neutron energy. The sum of the two energies then represents the full neutron energy. Kinematics similar to the Compton scatter process also restrict the neutron incident direction to be on a circle in the sky, and this circle may also pass through the location of the Sun. There are many other complicating reactions, particularly for neutrons, that do not yield accurate energies or incident

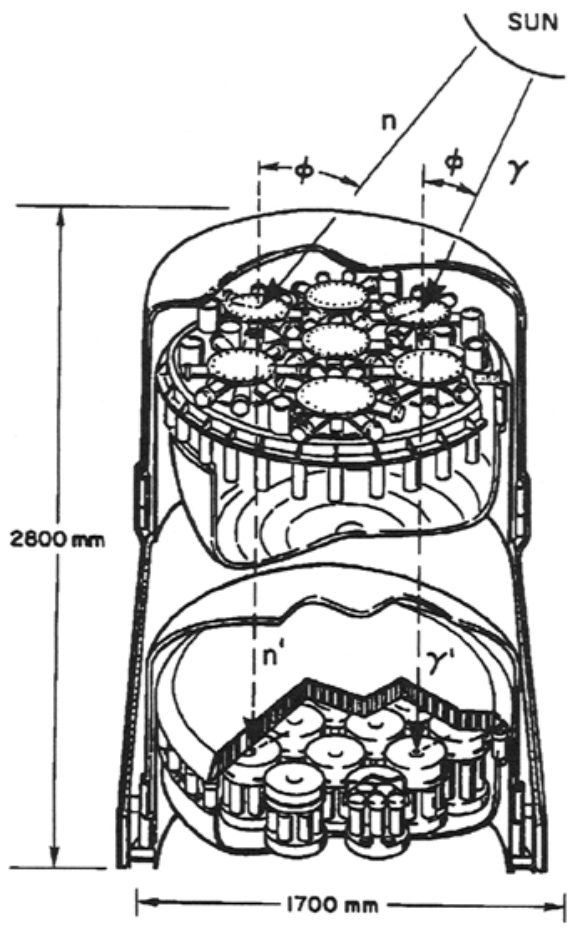

COMPTEL IMAGING COMPTON TELESCOPE

Figure 1. COMPTEL schematic. directions. The reader is referred to the instrument description by Schönfelder et al. (8) for more details.

\section{OBSERVATIONS}

\section{$\gamma$-ray and Neutron Images}

In general, the process of imaging greatly improves an instrument's signal-to-noise ratio, thereby enabling it to detect weaker signals and measure smaller fluxes. Such is the case with the COMPTEL. Exclusive of the science behind the flares that the Sun provides, the major feature of the COMPTEL solarflare measurements is their sensitivity, best illustrated with the observations of the 15 June 1991 solar flare. The image displayed in Figure 2 is that of the Sun in the time following the 


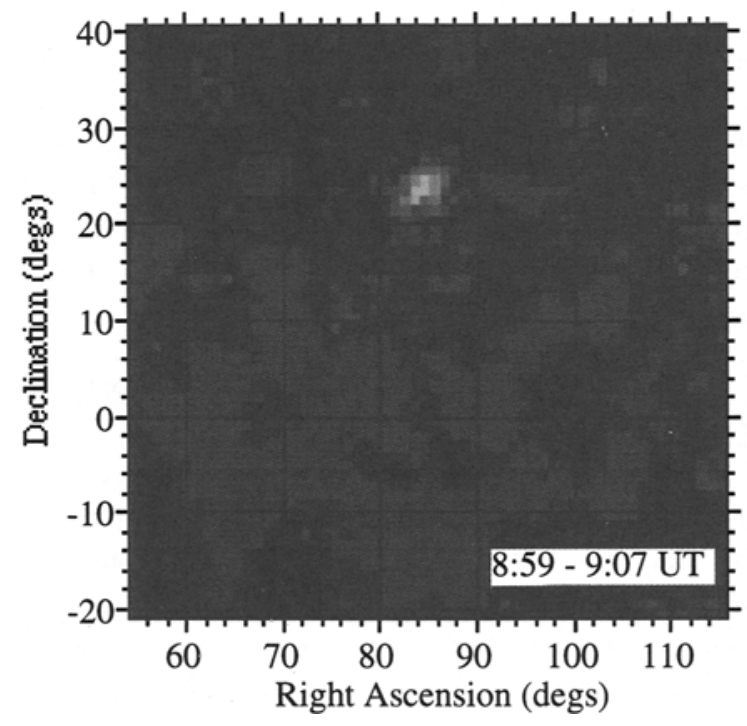

Figure 2. Gamma-ray image of the Sun from $0.8-8 \mathrm{MeV}$.

impulsive phase of a flare on 1991 June 15 (9). The image was obtained from only 9 minutes of data starting 30 minutes after the impulsive phase. Unfortunately, the resolution of the telescope does not permit resolving features smaller than $\sim 1^{\circ}$--twice the diameter of the Sun. The Observatory missed the impulsive phase of the flare during an occulted part of the orbit. However, the lingering low-level $\mathrm{MeV}$ radiation was still detectable primarily because of the imaging properties of COMPTEL.

The Sun also emitted neutrons during this flare and these particles were detected and measured by COMPTEL concurrent with the $\gamma$-ray measurements. Since 10-100 MeV neutrons are subrelativistic these neutrons arose from earlier epochs of the flare. The $\gamma$-ray imaging procedures were applied to the neutron data resulting in a neutron image of the Sun (Figure 3). The neutron flux from this flare was $30 \times$ smaller than that of the 3 June 1982 flare (10). We will return to this flare because it exhibits what we now call long-duration $\gamma$-ray emission, that is $\gamma$-ray emission persisting for $\sim 1$ hour or more after the impulsive phase of a flare.

\section{Neutron Measurements}

The long-sought-after ability to perform neutron spectroscopy has yielded new information that is difficult or impossible to obtain with $\gamma$-ray measurements. Long-duration high-energy flares have been a poorly measured and understood phenomenon before the Compton Observatory. One of the simplest of such flares to observe and measure was that of 9 June 1991. Here, the impulsive phase 
was observed near the beginning of a sunlit portion of a spacecraft orbit, enabling uninterrupted $\gamma$-ray and neutron measurements for more than 40 minutes after the impulsive phase. The subrelativistic nature of the $10-100 \mathrm{MeV}$ neutrons makes them arrive at the instrument mostly after the intense $\gamma$-ray flux has subsided. By measuring the energy of each registered neutron, we are able to trace the neutron back to its emission time at the Sun. A background-corrected and velocitycorrected intensity-time profile of these measured neutrons is shown in Figure 4. This $4 \sigma$ signal peaks well after the impulsive phase indicating that neutron emission is either delayed or of longer duration than that of the $\gamma$ rays. This conclusion is supported by the EGRET detection of $>50 \mathrm{MeV} \gamma$-ray emission at this time or later (6). Whereas the flux of $\mathrm{MeV}$ photons is declining monotonically after the impulsive phase, the neutron flux stays at an elevated level for at least 5 minutes. The evidence points to a clear hardening of the proton spectrum over the course of 5 minutes. This phenomenon was observed again two days later with the flare on 11 June 1991.

As mentioned above, the impulsive phase of the 15 June 1991 flare was unobservable with the Compton Observatory because of earth eclipse and SAA transit. However, again because of the subrelativistic nature of the sub-100 MeV neutrons, the neutron measurements provide information about the flare highenergy emissions in the occulted and blacked-out time periods. Debrunner et al. (10) concluded that the neutron emission was consistent with the high-energy $\gamma$ ray measurements made with the GAMMA-1 instrument $(11,12)$ that had more observing time on the Sun after the impulsive phase. The GAMMA-1 data show an exponentially declining flux of $>100 \mathrm{MeV} \gamma$ rays after the impulsive phase, similar to what was measured by COMPTEL in $\gamma$ rays (9).

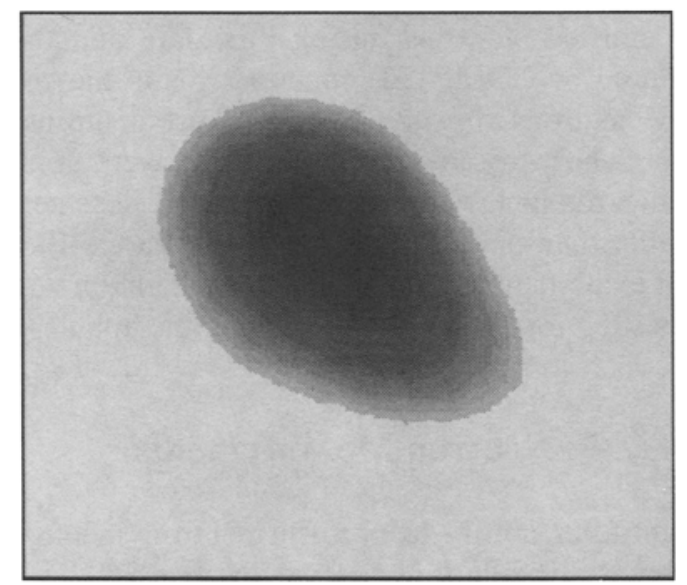

Figure 3. The Sun as it appears in neutron radiation. The image is $\sim 10^{\circ}$ in diameter and its asymmetrical shape is due to inaccuracies in the instrument point-spread function-the equivalent of optical distortion. 


\section{LONG-DURATION GAMMA-RAY FLARES}

Because COMPTEL has different types and areas of detecting elements it is capable of performing measurements at different epochs of the flare that have greatly different emission rates. In particular, the burst spectrometer can handle greater fluxes without encountering dead time effects, while the telescope can measure weaker fluxes later in a flare. Such is the case for the 11 June 1991 longduration, high-energy flare. In the impulsive phase of the flare the intense thermal $\mathrm{X}$-ray flux and the limited telemetry of the telescope limited the number of registered photons. However, the burst spectrometer measured the $0.6-10 \mathrm{MeV}$ spectrum every $12 \mathrm{~s} \mathrm{(2).} \mathrm{Later} \mathrm{in} \mathrm{the} \mathrm{flare} \mathrm{after} \mathrm{the} \mathrm{flux} \mathrm{subsided,} \mathrm{no} \mathrm{significant}$ measurements in the nuclear-line region were possible with the burst spectrometer while the telescope was collecting data at its maximum rate.

The 11 June 1991 solar flare is a bellweather event in solar high-energy physics. Its $\gamma$-ray emission $>50 \mathrm{MeV}$ persisted for more than 8 hours after the impulsive phase (13). Schneid et al. (6) also reported photon emission above 50 $\mathrm{MeV}$ minutes after the impulsive phase. The flare has attracted much attention since it tests our understanding of particle acceleration and transport. Opposing models of the particle behavior can be classified as to whether they revolve around the trapping of previously accelerated particles $(14,15)$ or the continuous acceleration of particles $(16,17)$. Distinguishing between these models is difficult with little more to go on than the $\gamma$-ray measurements.

COMPTEL measurements of the prolonged nuclear-line emission have been reported by Rank et al. (18) in these proceedings. The decay of the nuclear-line emission with respect to the emissions in the $2.223 \mathrm{MeV}$ line and the 8-30 MeV band provides a clue into the nature of the particle populations late in this event.

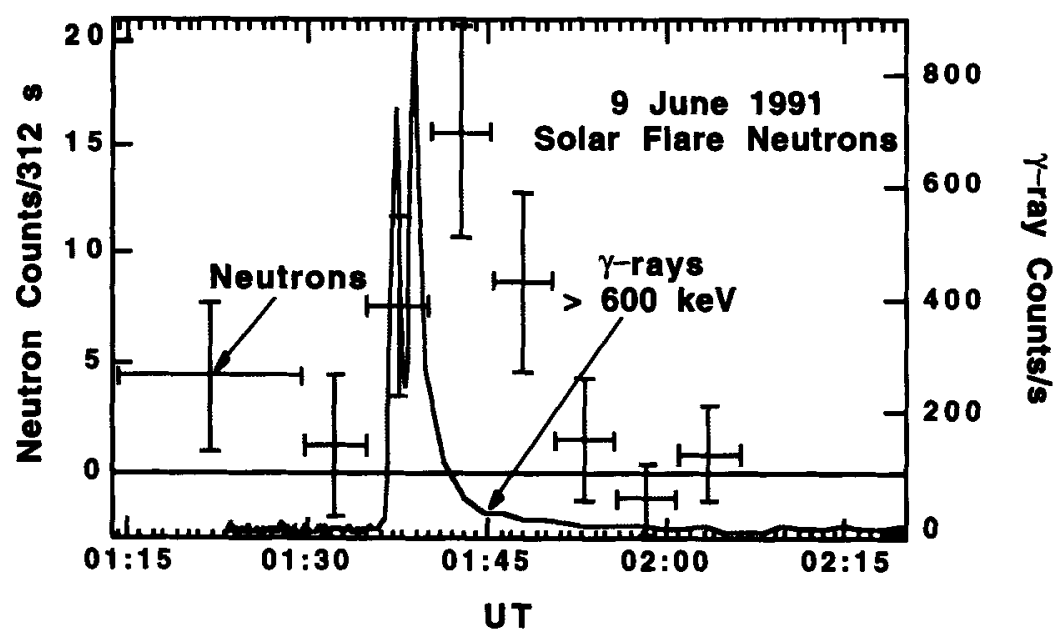

Figure 4. The velocity-corrected neutron emission from the 9 June 1991 flare. 


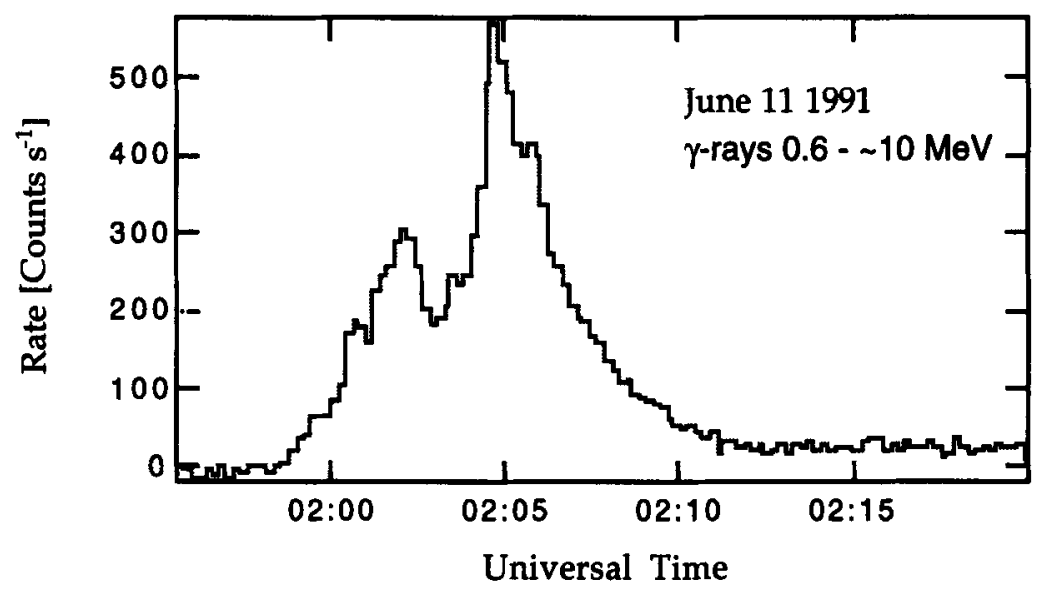

Figure 5. The background-corrected COMPTEL burst spectrometer count rate during the impulsive phase of the 11 June 1991 solar flare.

Rank et al. find that the behavior of these emission bands agree within statistics, supporting the hypothesis that the entire extended emission derives from protons, and that because of the constancy of the spectrum, the long lifetime of the proton population is due to prolonged acceleration rather than trapping. In the extended phase of the flare, the decay of all forms of $\gamma$-ray emission follows a double exponential decay-the first fast and the second much slower. The fast decay is on the order of 10 minutes and the slow decay is on the order of 100 minutes $(13,18)$.

The situation differs during the impulsive phase of the 11 June 1991 flare. Figure 5 shows the count rate of the COMPTEL burst spectrometer. The burst spectrometer accumulated 256 channel spectra every $12 \mathrm{~s}$ during the impulsive phase. The flare consisted of an impulsive phase that included two large bursts starting at approximately $0158 \mathrm{UT}$ and lasting for about 10 minutes. After about 0208 UT the flare as seen in $\gamma$ rays showed little structure-only a simple exponential decay after a possible local minimum occurring at 0212 UT. This change in tempo suggests that some fundamental change in the nature of the flare was taking place at this time and this may be reflected in the spectrum and species of particles being accelerated and the photons that those particles would subsequently produce. We can investigate this putative change with the COMPTEL burst spectrometer data.

A simple but useful measure of the general spectral shape of the protons responsible for the $\gamma$-ray emission is the ratio of the time-integrated flux of the 2.223 MeV line to that of the nuclear-line region of 4-7 MeV (19). With some care this ratio can be constructed for different phases of the flare (20). The difficulty in this analysis stems from the fact that the $2.223 \mathrm{MeV}$ deuterium formation line has a prolonged nature. The neutrons created by the energetic proton reactions require $\sim 100 \mathrm{~s}$ (density and ${ }^{3} \mathrm{He}$ dependent) to capture on 
hydrogen, resulting in an exponential decay of the $2.223 \mathrm{MeV}$ flux. The instantaneous $2.223 \mathrm{MeV}$ flux is thus due to the integrated effect of all neutron production prior to that moment.

One can see in Fig. 5 that after 0210 UT the count rate does not return to background. This excess is statistically significant and is mostly in the 2.223 $\mathrm{MeV}$ line. We interpret this as the start of the extended emission phase. We can compare the $2.223 \mathrm{MeV} / 4-7 \mathrm{MeV}$ flux ratio during the impulsive phase to that during the beginning of the extended phase to detect any change in the proton spectrum. Suleiman et al. (20) arbitrarily defined the interval 0158-0207 UT as the impulsive phase and the period $0210-0220 \mathrm{UT}$ as the so-called tail phase or the beginning of the extended phase of the flare. The gap between these intervals was excluded to reduce $2.223 \mathrm{MeV}$ cross-talk between the intervals. In the gap the $2.223 \mathrm{MeV}$ flux appears to be declining exponentially in magnitude. Interpolating this decay allows one to estimate the $2.223 \mathrm{MeV}$ flux lost from the impulsive phase interval and the excess flux introduced into the tail phase. (The corrections are both on the order of $30 \%$. The lines in the 4-7 MeV range are prompt and one can directly account for all their emission by simply integrating the flux over time.) Doing this and combining with the time-integrated fluxes in the range of 4-7 MeV yields a $2.223 \mathrm{MeV} / 4-7 \mathrm{MeV}$ flux ratio of $0.83 \pm 0.12$ for the impulsive phase and $4.7 \pm 2.0$ for the tail phase. The ratio in the tail phase could be larger than indicated by the error bars because so little flux is detected in the 4-7 MeV range. These ratios imply that the proton spectrum changes its shape significantly between the impulsive and tail phases of the flare. If the spectrum is of the form of a Bessel function, the hardness parameter $\alpha T$ changes from 0.02 to 0.12 , or if the spectrum is power law in shape the spectral index changes from -3.6 to $-2.3(21)$. Stated either way, the change represents a marked hardening of the proton spectrum responsible for the $\gamma$-ray emission. The major evolution of the occurs sometime around or before $0210 \mathrm{UT}$ when the proton spectrum hardens. Our value for the power-law index for the impulsive phase agrees with the value of -3.5 reported by Ramaty and Mandzhavidze (14), but our value for the tail phase represents a spectrum harder than what they concluded for the extended phase based solely on the high-energy spectrum hours after the impulsive phase. This disagreement in the extended or tail phases may not be significant because the two measures of the spectrum, i.e., the flux ratio and the shape of the photon spectrum $>100 \mathrm{MeV}$, span such a wide range of energy and different reaction channels that these simple measures may not be precise enough for such a comparison.

The 15 June 1991 solar flare in many ways resembles the 11 June 1991 flare. The two flares erupted in the same active region and both had prolonged highenergy photon radiation. Unfortunately, as mentioned above, the impulsive phase of the flare was unobservable to the Compton Observatory and the GAMMA-1 spacecraft as well. The similarity of the two flares may be more than just coincidental. Having originated in the same active region, they also may carry the 
signatures of the geometry and the topology of the region in their intensity-time profiles. Both models of prolonged $\gamma$-ray emission-trapping and continuous acceleration-are sensitive to the topology and dimensions of the magnetic structures containing the energetic particles. The time scales of the photon flux decay both follow the size of the active region, or more specifically, the size of the magnetic loops confining the particles. Rank et al. (18) have shown with the COMPTEL data that the time scales of the $2.223 \mathrm{MeV}$ emissions have within uncertainties the same long and short exponential decays. This suggests that the long-duration flare scenario played out in two events four days apart. Although the impulsive phases may have been different, the particle populations, or more specifically protons, that produced the prolonged emission both resided in the same loops or, at least, loops of approximately the same dimensions.

\section{CONCLUSIONS}

Although of considerable importance and interest, our measurements of longduration high-energy solar flares are largely based upon events stemming from a single active region in 1991. The specifics, as we saw, may depend on the details of the topology and dimensions of the particular region in which the flares occurred. Additional observations of flares in other active regions, supported by observations in other wavelength bands would broaden our knowledge base of this phenomenon. We must await the next solar maximum to study this further. The Compton Observatory and COMPTEL are both capable of operating and making good measurements in the next solar maximum. It is important that the mission be extended through the next maximum. Our experience in both solar physics and the operation of the instrument and some cooperation of the Sun will certainly yield data of the highest quality, thereby broadening our understanding of the enigmatic behavior of energetic particles in solar flares.

\section{ACKNOWLEDGMENTS}

We thank Raid Suleiman for help in preparing this paper. We also thank our COMPTEL colleagues and the staff at the Indiana University Cyclotron Facility for assistance in the neutron and gamma calibrations. This work was supported by NASA contract NAS5-26645.

\section{REFERENCES}

1. Hudson, H., and Ryan, J., Annu. Rev. Astron. Astrophys. 33, 239-82 (1995). 
2. Suleiman, R. et al., "COMPTEL'S Solar Flare Catalog," in High-Energy Solar Phenomena-A New Era of Spacecraft Measurements, 294, New York: AIP, (1994), pp. 5154.

3. McConnell, M., "An Overview of Solar Flare Results from COMPTEL," in High-Energy Solar Phenomena-A New Era of Spacecraft Measurements, AIP Conference Proceedings 294, New York: AIP, (1994), pp. 21-25.

4. Schwartz, R. A. et al., "BATSE Flare Observations in Solar Cycle 22," in The Compton Observatory Science Workshop, NASA Conference Publications 3137, NASA, (1992), pp. 457-468.

5. Murphy, R. J. et al., "OSSE Observations of Solar Flares," in Compton Symposium, AIP Conference Proceedings 280, New York: AIP, (1993), pp. 619-630.

6. Schneid, E. J. et al., "EGRET Observations of Extended High Energy Emissions from the Nuclear Line Flares of June 1991," in High-Energy Solar Phenomena-A New Era of Spacecraft Measurements, AIP Conference Proceedings 294, New York: AIP, (1994), pp. 94-99.

7. Dingus, B. L. et al., "EGRET Observation of the June 30 and July 2, 1991 Energetic Solar Flares," in High-Energy Solar Phenomena-A New Era of Spacecraft Measurements, AIP Conference Proceedings 294, New York: AIP, (1994), pp. 177-179.

8. Schonfelder, V. et al., Ap. J. Suppl. 86, 657-692 (1993).

9. McConnell, M. et al., Adv. Sp. Res. 13, 245-248 (1993).

10. Debrunner, $H$. et al., "Neutrons from the 15 June 1991 solar flare," in 23rd International Cosmic Ray Conference, 3, (1993), pp. 115-118.

11. Akimov, V. V. et al., "Some Evidences of Prolonged Particle Acceleration in the HighEnergy Flare of June 15, 1991," in High-Energy Solar Phenomena-A New Era of Spacecraft Measurements, 294, New York: AIP, (1994), pp. 106-111.

12. Akimov, V. V. et al., "The high-energy gamma-ray flare of June 15, 1991: Some evidences of prolonged particle acceleration at the post-eruption phase," in Proc. of Kofu Symposium, NRO Report \#360, Nobeyama Radio Observatory, (1994), pp. 371-374.

13. Kanbach, G. et al., Astron. and Astrophys. Suppl. 97, 349-353 (1993).

14. Ramaty, R., and Mandzhavidze, N., "Theoretical Models for High Energy Solar Flare Emissions," in High-Energy Solar Phenomena-A New Era of Spacecraft Measurements, AIP Conference Proceedings 294, New York: AIP, (1994), pp. 26-44.

15. Mandzhavidze, N., and Ramaty, R., Ap. J. 389, $739-755$ (1992).

16. Ryan, J. M., and Lee, M. A., Ap. J. 368, 316-324 (1991).

17. Ryan, J. M., Bennett, E., and Lee, M. A., "Proton Acceleration in Long Duration Flares," in Advances in Solar Physics, 432, Berlin: Springer-Verlag:, (1994), pp. 273-278.

18. Rank, G. et al., "Extended $\gamma$-Ray Emission in Solar Flares," in these proceedings, New York: AIP, (1996).

19. Hua, X.-M., and Lingenfelter, R. E., Solar Phys. 107, 351-383 (1987).

20. Suleiman, R. M. et al., Bull. Am. Astron. Soc. 27, 987 (1995).

21. Hua, X.-M., and Lingenfelter, R. E., Ap. J. 323, 779-794 (1987). 